\title{
ON CLOSED TWISTED CURVES
}

\author{
SUELI I. RODRIGUES COSTA
}

(Communicated by Jonathan M. Rosenberg)

\begin{abstract}
Twisted curves in $\mathbf{R}^{\prime \prime}$ are those which have independent derivatives up to order $m$. For plane and spatial curves the twisted condition is equivalent to never vanishing curvature and torsion respectively. We give a necessary and sufficient condition for a $(q, p)$-curve on a torus to be twisted, and use those curves to construct closed twisted curves in $\mathbf{R}^{m}$ for all $m$.
\end{abstract}

\section{INTRODUCTION}

The concept of order $m$ singularity which involves higher order derivatives was introduced by E. Cartan and has been developed by several authors. Important references on this subject are [16] and [5], where standard notions and results such as tangent bundle, immersion, and the Whitney theorem are extended to order $m$ and some relationships with differential geometry are described.

For curves in $\mathbf{R}^{n}$ the $m$-tangent space at a point is generated by its derivatives up to order $m$. If these vectors are linearly dependent at some point, we have a singularity of order $m$. Straight lines are only nonsingular of order 1. Plane curves are nonsingular of order 2 if and only if they have nowhere vanishing curvature. Points of null torsion are third order singularities for curves in space.

Likewise, an order $m$ singularity of a curve in $\mathbf{R}^{m}$ is a point where it fails to "curve" sufficiently. This idea can be stated precisely using higher order curvatures. Order $m$ nonsingular curves in $\mathbf{R}^{m}$ are those having nowhere vanishing curvature of order $m-1$, and we may call them twisted.

The four-vertex theorem in its spatial version [2] shows that simple closed nonvanishing torsion curves lying on strictly convex surfaces in space do not exist. This leads us to look for such curves, for example, spiraling on a torus. The $(q, p)$-curve spirals around the torus $p$ times in the "vertical" sense while spiraling $q$ times in the "horizontal" sense. We show (Proposition 1) that the $(q, p)$-curve on a standard torus with radii in proportion to $a>1$ has

Received by the editors November 3, 1988 and, in revised form, April 17, 1989.

1980 Mathematics Subject Classification (1985 Revision). Primary 53A04; Secondary 57R99.

Key words and phrases. Nowhere vanishing torsion curves, four-vertex theorem, order $n$ singularity.

The author was supported in part by CNPq-Brazil, 20.0162-84. 
nonvanishing torsion if and only if for $n=\frac{p}{q}$, we have $n^{2}>1$ and $\frac{2 n^{2}+1}{n^{2}-1}<$ $a<n^{2}+1$.

There exist only four classes of homotopy among closed curves of nonvanishing torsion in $\mathbf{R}^{3}$ [13]. For the curves on the torus described above, the representatives of these classes are given by $n$ positive or negative and by even or odd $p+q$.

For even values of $m$, closed twisted curves in $\mathbf{R}^{m}$ (and with constant curvature up to $m-1$ ) arise naturally as geodesics on flat tori. For odd values of $m$, closed curves with constant nonvanishing curvatures do not exist [1]. Starting from curves with nonvanishing torsion on a torus, we give a construction of closed twisted curves in $\mathbf{R}^{m}$ for odd $m(\S 2)$.

\section{Closed twisted CURVes in SPACE}

We consider here $C^{4}$ curves $\mu: I \rightarrow \mathbf{R}^{3}$ such that their derivatives $\mu^{\prime}$ and $\mu^{\prime \prime}$ are everywhere linearly independent, and we denote by $\kappa$ and $\tau$ the associated curvature and torsion. For the Frenet moving frame of $\mu,\left\{v_{1}, v_{2}, v_{3}\right\}$, the usual equations $v_{3}^{\prime}=\tau v_{2}$ and $\tau=\operatorname{det}\left[\mu^{\prime}, \mu^{\prime \prime}, \mu^{\prime \prime \prime}\right] \cdot\left|\mu^{\prime} \times \mu^{\prime \prime}\right|^{-2}$ express the equivalence between the three conditions: stationary osculating plane, null torsion, and third order singularity.

Searching $\mathbf{R}^{3}$ for examples of closed curves with nonvanishing torsion, we found that they were not obvious and references to them are minimal and not explicit. The most interesting fact is that obstructions to the existence of such curves are related to convexity.

The four-vertex theorem in its spatial version states that "a simple closed curve lying on a strictly convex surface has at least four points of vanishing torsion (vertices)." A relationship between this theorem and its well-known plane version was already established by A. Kneser in 1912 [12]: The stereographic image of a plane curve vertex $\left(\kappa^{\prime}=0\right)$ is a point of null torsion for the spheric curve. The version for strictly convex surfaces (met by any line in at most two points) was stated by $\mathrm{H}$. Morhmann in 1917 [15], the first complete proof being given in 1958 by M. Barner and F. Flohr [3].

Other related results have been stated for simple closed curves: "If the curve meets no plane in more than four points, it has exactly four vertices" ( $P$. Scherk and B. Segre in [20]). Having at least four vertices is also implied for each of the following two conditions: (i) through each two points of the curve there is a plane not meeting it elsewhere (M. Barner [2]) and (ii) there is no pair of parallel tangents (B. Segre [21]). No trisecants and no cross-tangents implies two vertices (S. Jones [10]). It is a conjecture (P. Scherk) that the four-vertex theorem is valid for convex curves (lying on the boundary of their convex hulls). J. Bisztriczky [4] showed that this is true for inflexional curves having no trisecants and meeting any plane in a finite number of points. A generic version for that is to be found in [19]. 
The convex barrier leads us to look for twisted closed curves spiraling on a torus. Consider the standard torus in space obtained by rotating a unit-radius circle around an axis, the distance of which to the center is $a>1$. The $(q, p)$ curve spins $p$ times in the vertical sense and $q$ times in the horizontal sense. In cylindrical coordinates this is given by $r=a+\cos (n \theta), z=\sin (n \theta)$, where $n=p / q$ and $\theta$ varies in an interval of length $2 \pi q$. This can also be given in Cartesian coordinates by

$$
\begin{aligned}
\alpha(t)= & \left(a \cos t+\frac{1}{2} \cos (n+1) t+\frac{1}{2} \cos (n-1) t,\right. \\
& \left.a \sin t+\frac{1}{2} \sin (n+1) t-\frac{1}{2} \sin (n-1) t, \sin t\right) .
\end{aligned}
$$

We remark that these are simple closed curves and are knotted whenever $q \neq 1$ and $p$ and $q$ are coprime.

Analyzing the torsion of those curves (see also Figures 1 and 2) we obtain the following:

Proposition 1. The $(q, p)$ curve on a standard torus in space with radii in proportion to $a>1$ is of nonvanishing torsion if and only if for $n=p / q$ we have $n^{2}>1$ and

$$
\frac{2 n^{2}+1}{n^{2}-1}<a<n^{2}+1
$$

As a direct consequence of this result we have the following:

(i) Only on a thin torus $(a>2)$ is it possible to find $n$ such that the $(1, n)$ curve (or $(q, p)$-curve) has nonvanishing torsion.

(ii) There exists $a(q, p)$-curve with nonvanishing torsion on some conveniently proportioned torus if and only if we have $\left(\frac{p}{q}\right)^{2}>1+\sqrt{3}$.

Proof. The proof of Proposition 1 is carried out by direct calculation. We take the matrix $M(t)$ whose columns are the coordinate vectors $\alpha^{\prime}, \alpha^{\prime \prime}$, and

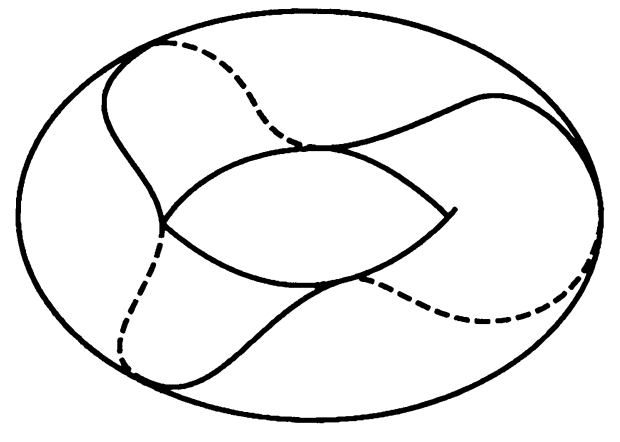

Figure 1. The (1,3)-curve on a torus is nonvanishing torsion for $\frac{19}{8}<a<10$.

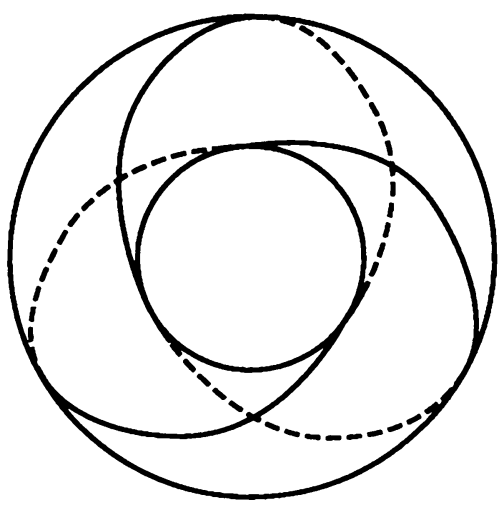

Figure 2. The (2, 3)-curve (trefoil) on any torus always has points of null torsion. 
$\alpha^{\prime \prime \prime}$ with respect to the appropriate moving frame $v_{1}=(\cos t, \sin t, 0), v_{2}=$ $(-\sin t, \cos t, 0), v_{3}=(0,0,1)$. Operating successively with line-equivalence in $M(t)$, we conclude that the condition of linear dependence of $\alpha^{\prime}, \alpha^{\prime \prime}$, and $\alpha^{\prime \prime \prime}$ occurs if and only if $x=\cos n t$ is a root of the polynomial

$$
P(x)=-\left(n^{2}-1\right) x^{3}+2 a\left(2 n^{2}+1\right) x^{2}+\left[2 n^{2}\left(n^{2}+2\right)-a^{2}\left(n^{2}-1\right)\right] x-a n^{2}\left(n^{2}+2\right) .
$$

Analyzing the growth of this polynomial for $-1 \leq x \leq 1$, we can complete the proof by following these three steps:

(i) $P(0)=-a^{2} n^{2}\left(n^{2}+2\right)<0$, for all $n$ and all $a$;

(ii) $P(1)=\left(1-n^{2}\right) a^{2}+\left(-n^{4}+2 n^{2}+2\right) a+2 n^{4}+3 n^{2}+1$, and so $P(1)>0$ for $n^{2} \leq 1$;

(iii) for $n^{2}>1$, checking the roots and signs of $P(1)$ and $P(-1)$, we see that $\frac{2 n^{2}+1}{n^{2}-1}<a<n^{2}+1$ is a necessary condition for $P(x)$ having no roots for $-1 \leq x \leq 1$.

To verify its sufficiency we observe first that in this case, $P^{\prime}(x)$ always has two roots, $x_{1}<x_{2}$ and $x_{2}>1$ (see Figure 3). As $x_{1}$ and $x_{2}$ are respectively local minimum and maximum for $P(x)$, the highest value for $P(x),-1 \leq x \leq 1$, will be $P(-1)$ or $P(1)$. Since in this case $P(-1)<0$ and $P(1)<0, P(x)$ is never zero in this interval.

Remarks. Here are some remarks.

(i) For $n=1$ we have the diagonal curve which has four, three, or two points of null torsion according to $a>2$ (thin tori), $a=2$, or $a<2$;

(ii) Proposition 1 is still valid for irrational $n$ (open dense curves) and for $0<a \leq 1$ (tori with self-intersection).

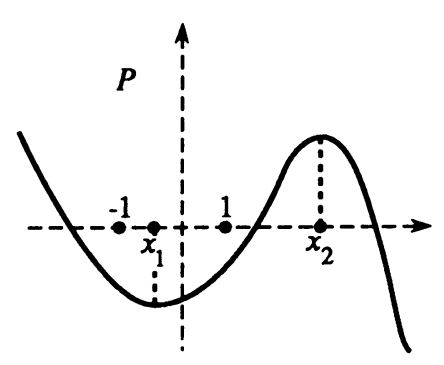

FiguRE 3. 
As mentioned before, bibliographic references to closed curves with nonvanishing torsion are scarce. One merit of Proposition 1, therefore, is that it provides a class of explicit algebraic examples. Whether there exist algebraic closed curves with constant torsion is still an open question [22]. Reference [8] deals with the number $n(\alpha)$ of triply tangent planes to a curve with nonvanishing torsion and makes a unique explicit reference to such curves as the $(2,3)$-curve on a properly proportioned radii torus which has $n(\alpha)=2$. According to our result, this curve always has points of null torsion since $\left(\frac{3}{2}\right)^{2}<1+\sqrt{3}$. Could it be deformed slightly in order to be twisted and still have $n(\alpha)=2$ ? We believe that the $(1,3)$-curve, although unknotted, can serve the same purpose. What about the other $(q, p)$-curves?

Deformations. We may consider deformations of closed space curves preserving the independence of their derivatives, namely the first, second, and third order homotopy (through immersions) and isotopy (through embeddings) classification. For the first order, we have just one homotopy class (H. Whitney) and the isotopy classification is a central problem of knot theory. For the second order, there are two homotopy classes of closed curves with nonvanishing curvature (E. Feldman [6]) and the isotopy classes are determined by the knot class plus the self-linking number (W. Pohl and J. Dillon, unpublished, see [17] and [18]). For the third order, there are four homotopy classes of closed curves with nonvanishing torsion (J. Little [13]) and the isotopy classification is still open, although it has been solved for a large class of curves. It is conjectured that the same condition for second order isotopy applies (J. Little [14]).

For the $(q, p)$-curves on torus satisfying Proposition 1 , the representatives of the four homotopy classes are given by positive or negative $n$ and by even or odd $p+q$. This can be seen by analyzing their spherical tangent indicatrices. As the original curves have nonvanishing torsion, they must have a double point [7]. Using the symmetry of the $(q, p)$-curve (a $\frac{2 \pi q}{p}$ sector rotated $p$ times), we see (Figure 4) that the projection of its spherical tangent indicatrices on the $x y$ plane is a curve with rotation number $p+q$ and will fit the four classes in [13], as stated above. Finally, we remark that among the curves given by Proposition 1 we have infinitely many self-linking numbers.

\section{Closed twisted curves in $\mathbf{R}^{\prime \prime}$}

Simple examples of twisted curves in $\mathbf{R}^{m}$ are given by open curves like $\left(t, t^{2}, \ldots, t^{m}\right)$. The complex curve $\left(z, z^{2}, \ldots, z^{m / 2}\right)$ will induce closed twisted curves for even $m$, but as in $\mathbf{R}^{3}$, we cannot find such natural examples for odd values of $m$.

The equivalence between third order singularity and null torsion can be extended using higher order curvatures (see [9] and [11]). For $C^{m+1}$ curves which have linearly independent derivatives up to order $m-1$, the moving Frenet frame is constructed as it is in usual space using the Gram-Schmidt process. We get orthonormal vectors $E_{1}, \ldots, E_{m-1}$ and add the last vector as the unit 

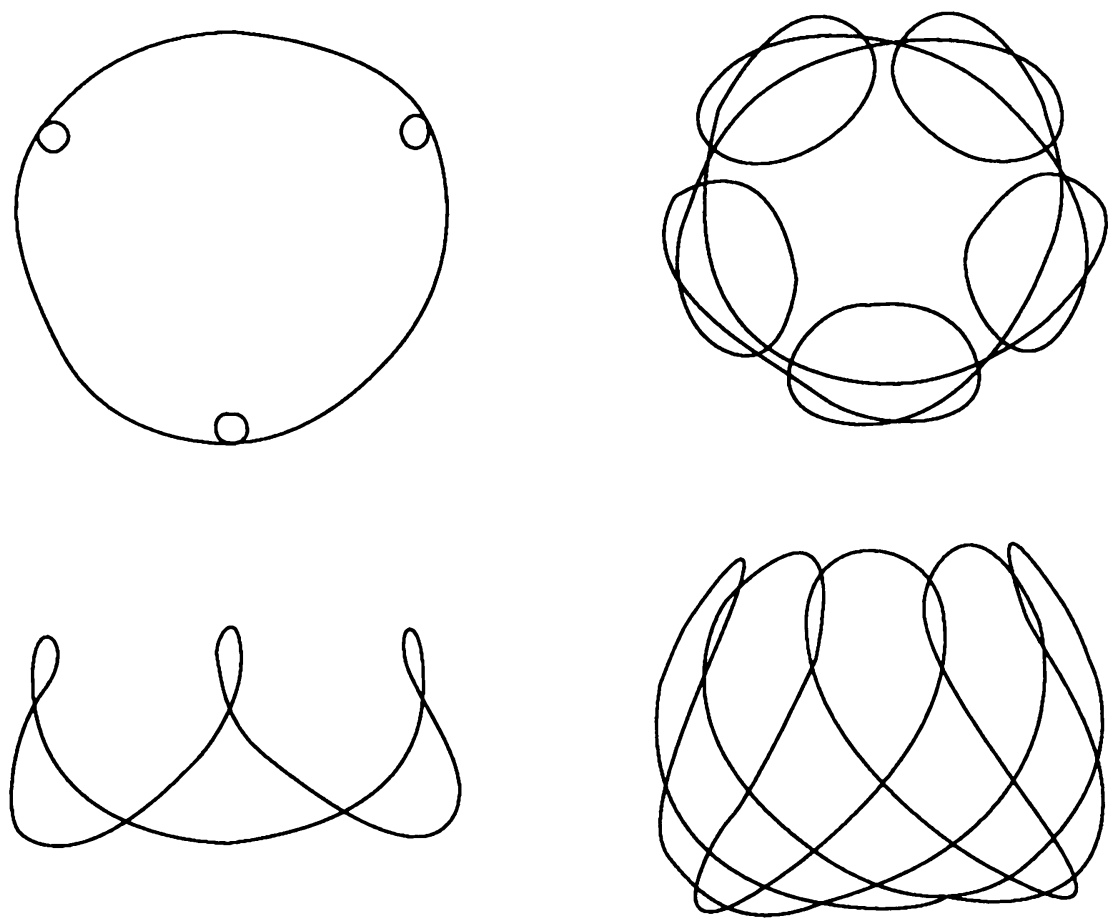

FIGURE 4. Spherical tangent indicatrices of $(q, p)$-curves on tori. On the left we have $p=3, q=1$, and $a=7$ (upper and frontal views). On the right, $p=5, q=2$, and $a=2.8$.

vector in $\mathbf{R}^{m}$ such that $\left\{v_{1}, \ldots, v_{m}\right\}$ has a positive orientation. The $i$ th curvature is defined as the mapping $\kappa_{i}=\left\langle v_{i}, v_{i+1}\right\rangle /\left|\alpha^{\prime}\right|$ for $i=1, \ldots, m-1$, and the Frenet equations are stated:

$$
\left[\begin{array}{c}
v_{1}^{\prime} \\
\vdots \\
v_{n}^{\prime}
\end{array}\right]=\left|\alpha^{\prime}\right|\left[\begin{array}{cccc}
0 & \kappa_{1} & \cdots & 0 \\
-\kappa_{1} & 0 & \cdots & 0 \\
0 & -\kappa_{2} & \cdots & \kappa_{m-1} \\
\vdots & & & \vdots \\
0 & \cdots & -\kappa_{m-1} & 0
\end{array}\right]\left[\begin{array}{c}
v_{1} \\
\vdots \\
v_{n}
\end{array}\right]
$$

For curves $\alpha: I \rightarrow \mathbf{R}^{m}$ with linearly independent $\alpha^{\prime}, \ldots, \alpha^{m-1}$, singularity of order $m$ at a point corresponds to the vanishing of $\kappa_{m-1}$ at this point. This can be verified by differentiating the initial relationship $\alpha^{\prime}=\left|\alpha^{\prime}\right| v_{1}$ and by using the Frenet equations in order to obtain

$$
\operatorname{det}\left[\alpha^{\prime}, \alpha^{\prime \prime}, \ldots, \alpha^{(m)}\right]=\left|\alpha^{\prime}\right|^{\frac{m(m+1)}{2}} \cdot \kappa_{1}^{m-1} \cdots \kappa_{m-2}^{2} \cdot \kappa_{m-1} .
$$

For even $m=2 \kappa$ we can take the geodesics on flat tori $\alpha(t)=\left(a_{1} \cos p_{1} t\right.$, $\left.a_{1} \sin p_{1} t, \ldots, a_{k} \cos p_{k} t, a_{k} \sin p_{k} t\right)$. The determinant of the matrix $M(t)$, the 
columns of which are the derivatives of $\alpha$ up to order $m$, is

$$
\operatorname{det} M=D \cdot \prod_{j>i}^{k}\left(p_{j}^{2}-p_{i}^{2}\right)^{2}, \quad D \neq 0,
$$

which is constant. So the curve is twisted if and only if $p_{i} \neq p_{j}$ for all $i$ and $j$. Moreover, using the relationship $\kappa_{i}=\frac{\left|E_{i+1}\right|}{\left|E_{i}\right| \cdot\left|E_{1}\right|}$, we can see that all the curvatures up to order $m-1$ are constant.

For odd dimension, curves with constant nonvanishing curvatures cannot be closed. As is shown in [1], those curves must be helices $\beta(t)=(\alpha(t), a t+b)$ where $\alpha(t)$ is as above. That is why, as in $\mathbf{R}^{3}$, closed twisted curves are not given in such a natural way in odd dimensional spaces. Nevertheless, they could be obtained from curves described in Proposition 1 by a reduction process as follows:

Lemma. The curve $(\alpha(t), \cos p t, \sin p t)$ in $\mathbf{R}^{\prime \prime}$ is twisted if and only if $p^{2} \alpha+\alpha^{\prime \prime}$ is twisted.

Proposition 2. For $m=3+2 \kappa$ and rational $p_{1}, \ldots, p_{k}$ with $p_{i} \neq p_{j}$, there exists a curve $u$ in $\mathbf{R}^{3}$ such that $\alpha(t)=\left(u(t), \cos p_{1} t, \sin p_{1} t, \ldots, \cos p_{k} t\right.$, $\left.\sin p_{k} t\right)$ is a simple closed twisted curve.

$u(t)$ can be chosen as a $(q, p)$-curve on an elliptic torus, where $n=\frac{p}{q}$ satisfies Proposition 1 .

The statement of the lemma is given by the calculations of the order $m$ determinant of the derivatives up to order $m$ through line-equivalence and the generalized Laplace rule. The proof of Proposition 2 is carried out by induction on $\kappa$. The curve $u$ is obtained by recursion on the differential operator of the lemma $\beta=\left[p_{k}\right] \cdots\left[p_{1}\right](u)(*)$, where $\left[p_{j}\right](\alpha)=p_{j}^{2} \alpha+\alpha^{\prime \prime}$ and $\beta$ must be a twisted curve in space. We observe that if we choose $\beta$ as a $(1, n)$-curve on a torus and restrict ourselves to the class of curves

$$
\begin{aligned}
u(t)= & (A \cos t+B \cos (n+1) t+C \cos (n-1) t, \\
& E \sin t+F \sin (n+1) t+G \sin (n-1) t, H \sin n t),
\end{aligned}
$$

where $n \neq \pm p_{i}$ and $n \neq \pm p_{i} \pm 1, n^{2}>1+\sqrt{3}, p_{i} \neq \pm 1$, then $(*)$ has a solution $u$ in the same class.

As an example, we get $\beta(t)=(u(t), \cos 4 t, \sin 4 t)$ twisted in $\mathbf{R}^{5}$ choosing $n=2$ and $a=4$, where

$$
u(t)=\left(\frac{3}{10} \cos t+\frac{1}{4} \cos 3 t, \frac{7}{30} \sin t+\frac{1}{14} \sin 3 t, \frac{1}{12} \sin 2 t\right) .
$$

Here $u(t)$ is a $(1,2)$-curve on an elliptic torus.

\section{THE FOUR-VERTEX THEOREM FOR CURVES ON A CYLINDER}

We consider closed curves on a standard cylinder in space given by $\alpha(t)=$ $(f(t), \cos p t, \sin p t)$, where $f$ is a $C^{4}$ real periodic function with period $2 \pi$. 


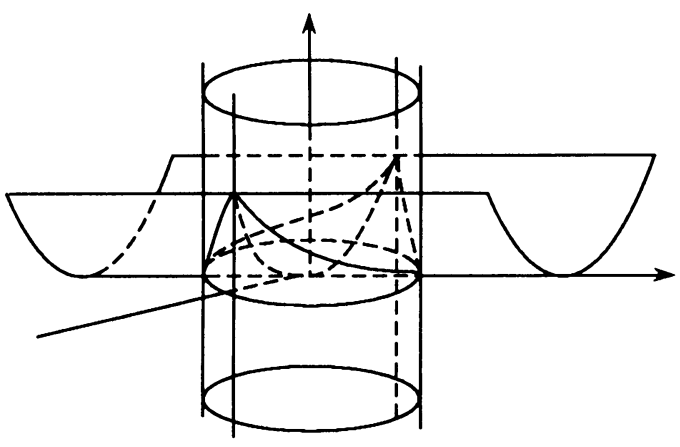

FIgURE 5. A four-vertex curve on a cylinder.

We note that these curves are not the kind covered by the four-vertex versions (see Figure 5) we listed in $\S 2$.

It is a consequence of our lemma for $m=3$ that they have at least two vertices, since $p^{2} f+f^{\prime \prime}$ as a periodic function has at least two critical points in its period. For $p=1$, we have simple graphic curves and the M. Barner condition applies. The previous lemma reduces the four-vertex theorem for these curves to the following statement:

For every real periodic $C^{4}$ function $f$ of period $2 \pi, f^{\prime \prime \prime}+f^{\prime}$ has at least four zeros in this period.

Proof. We give a proof of this result using the induced real function of a complex variable defined on the circle $S^{1}$. This proof is carried out according to the following sketch:

(i) If $\int_{S^{1}} g(z) d s=0$ and $g$ has at most three zeros in $S^{1}$, then $\int_{S^{\prime}} z g(z) d s \neq 0$.

The underlying idea here is that of mass center (suggested to me by D. Sullivan). As we have at most three zeros, the circle is divided into at most two intervals, where $g$ is positive $\left(R_{1}\right)$ and negative $\left(R_{2}\right)$. See Figure 6. If we think of $|g(z)|$ as a density function, we have $\int_{S^{\prime}} z g(z) d s=2 \pi\left(C_{1}-C_{2}\right)$, where $C_{1}$ and $C_{2}$ are the mass centers, of $R_{1}$ and $R_{2}$ respectively. So this integral cannot be zero.

(ii) As $g=f^{\prime}+f^{\prime \prime \prime}$ satisfies $\int_{S^{\prime}} g(z) d s=0$ and $\int_{S^{\prime}} z g(z) d s=0$ (from the Fourier series expansion of $f$ we see that the series of $g(s) \cos s$ and $g(s) \sin s$ do not have constant terms), we use (i) to conclude that $g=f^{\prime}+f^{\prime \prime \prime}$ must have at least four roots.

The relationship between higher order singularities and the global geometry of manifolds is by far the most interesting subject related to this article. It 


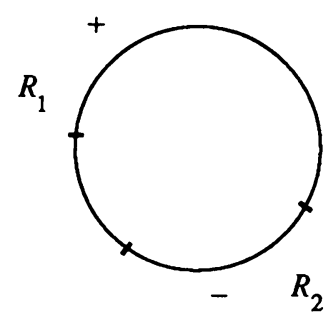

Figure 6.

is very possible that the convexity relations, which are given for curves by the four-vertex theorem, can be extended to manifolds of higher dimension.

\section{ACKNOWLEDGMENTS}

The author wishes to thank A. Conde, M. do Carmo, W. Pohl, and D. Sullivan for helpful conversations, and the Institute for Advanced Study, Princeton, where part of this work was done.

\section{REFERENCES}

1. J. L. Barbosa and M. do Carmo, Helicoids, catenoids, and minimal hypersurfaces of $\mathbf{R}^{n}$ invariants by l-parameter, An. Acad. Brasil. Ciênc. 53 (1981), 403-408.

2. M. Barner, Uber die Mindestanzahl stationarer Schmiegebenen bei geschlossenen strengkonvexen Raumkurven, Abh. Math. Sem. Univ. Hamburg 20 (1956), 196-215.

3. M. Barner and F. Flohr, Der Vierscheitesatz und seine Verallgemeinrugen, Der Mathematikunterricht 44 (1958), 43-73.

4. T. Bisztriczky, Inflexional convex space curves, Canad. J. Math. 36 (1984), 537-545.

5. E. A. Feldman, The geometry of immersions I, Trans. Amer. Math. Soc. 120 (1965), 185223.

6. __ Deformations of closed space curves, J. Differential Geom. 2 (1968), 67-75.

7. W. Fenchel, On the differential geometry of closed space curves, Bull. Amer. Math. Soc. $\mathbf{5 7}$ (1951), 44-54.

8. M. H. Freedman, Planes triply tangent to curves with nonvanishing torsion, Topology 19 (1980), 1-8.

9. H. Gluck, Higher curvatures of curves in euclidean space, Amer. Math. Monthly 73 (1966), 699-704.

10. E. S. Jones, A generalization of the two-vertex theorem for space curves, J. Differential Geom. 10 (1975), 1-10.

11. W. Klingenberg, A course in differential geometry, Springer-Verlag, New York, 1977.

12. A. Kneser, Bem erkungen über die Anzahl der Extreme der Krümmung geschlossenen kurven und über verwandte Fragen in einer nicht-euklidischen geometrie, Festschrift $\mathrm{H}$. Weber, 1912, pp. 170-180.

13. J. A. Little, Third order nondegenerate homotopies of space curves, J. Differential Geom. 5 (1971), 503-515.

14. __ Space curves with positive torsion, Ann. Mat. Pura Appl. (4) 116 (1978), 57-86.

15. H. Mohrmann, Die minimalzahl der stationären Ebenen eines räumlichen ovals, Sitz Ber Kgl Bayerichen Akad. Wiss. Math. Phys. Kl. (1917), 1-3. 
16. W. F. Pohl, Differential geometry of higher order, Topology 1 (1962), 170-211.

17. _ The self-linking number of a closed space curve, J. Math. Mech. 17 (1968), 975-986.

18. __ Singularities in differential geometry of submanifolds, Proc. Liverpool Singularities Sympos. II, Lecture Notes in Math., no. 209, Springer-Verlag, New York, 1971.

19. M. C. Romero-Fuster, Convexly-generic curves in $\mathbf{R}^{3}$, Geom. Dedicata 28 (1988), 7-29.

20. P. Scherk, Über reele geschlossene Raumkurven vierter Orduung, Math. Ann. 112 (1936), 743-766.

21. B. Segre, Alcune propietà differenziale in grande delle curve chiuse sghembe, Rend. Mat. Serie VI, 1 (1968), 237-297.

22. J. L. Werner, Closed curves of constant torsion II, Proc. Amer. Math. Soc. 67 (1977), 306-308.

Instituto de Matemática, Universidade de Campinas, 13081 Campinas SP, Brazil 